\title{
A Study of Numerical Schemes for Incompressible Fluid Flows
}

C.M. OISHI, J.A. CUMinATO, V.G. FERREIRA, M.F. TOMÉ, A. CASTELO, Departamento de Computação e Estatística, ICMC, USP, 13560-970 São Carlos, SP, Brasil

N. MANGIAVACCHI, Departamento de Engenharia Mecânica, Universidade do Estado do Rio de Janeiro, UERJ, 20550-900 Rio de Janeiro, RJ, Brasil.

\begin{abstract}
The present work is concerned with a study of numerical schemes for solving two-dimensional time-dependent incompressible free-surface fluid flow problems. The primitive variable flow equations are discretized by the finite difference method. A projection method is employed to uncouple the velocity components and pressure, thus allowing the solution of each variable separately (a segregated approach). The diffusive terms are discretized by Implicit Backward and CrankNicolson schemes, and the non-linear advection terms are approximated by the high order upwind VONOS (Variable-Order Non-oscillatory Scheme) technique. In order to improved numerical stability of the schemes, the boundary conditions for the pressure field at the free surface are treated implicitly, and for the velocity field explicitly. The numerical schemes are then applied to the simulation of the Hagen-Poiseuille flow, and container filling problems. The results show that the semi-implicit techniques eliminate the stability restriction in the original explicit GENSMAC method.
\end{abstract}

\section{Introduction}

In many fluid flow problems, the viscous forces are dominant and several numerical techniques have been developed for the solution of this class of flows. In these problems, the Reynolds number is often much smaller than 1 . Due to this fact, numerical techniques that apply an explicit formulation, as GENSMAC (GENeralized Simplified Marker-And-Cell) method [11], introduce the parabolic stability restriction, making the time step very small for some applications, justifying the need for methods with better stability properties like implicit schemes. Authors such as [1], [3], [8] and [10] show an overview about implicit methods for Navier-Stokes equations. In the present paper, it is proposed a modification in the explicit GENSMAC method by adding implicit schemes and treating the boundary conditions for the pressure field at the free surface implicitly. The time-marching producere is based on the projection methods (see, for example, [5] and [7]). The methods are based on a staggered grid system and they solve the full Navier-Stokes equations 
in primitive variables. In particular, the methods solve confined and free surface flows.

In non-dimensional conservative form, the equations for incompressible viscous newtonian flows are

$$
\begin{gathered}
\frac{\partial \mathbf{u}}{\partial t}+\nabla \cdot(\mathbf{u u})=-\nabla p+\frac{1}{R e} \nabla^{2} \mathbf{u}+\frac{1}{F r^{2}} \mathbf{g}, \\
\nabla \cdot \mathbf{u}=0,
\end{gathered}
$$

where $t$ is time, $\mathbf{u}=[u(x, y, t), v(x, y, t)]$ is the velocity vector field, $p=p(x, y, t)$ is pressure per unit of mass and $\mathbf{g}=\left(g_{x}, g_{y}\right)$ is the gravity field. The non-dimensional parameters $R e=L U / \nu$ and $F r=U / \sqrt{g L}$ are the Reynolds and Froude numbers, respectively, being $L$ and $U$ the length and the velocity scales, and $\nu$ is the kinematic viscosity coefficient of the fluid.

\section{Numerical Method}

The numerical method proposed in this work to solve the Equations (1.1) and (1.2) is basically a modification of the GENSMAC method. Firstly, a provisional velocity field $\tilde{\mathbf{u}}$ is calculated from Equation (1.1), that is,

$$
\frac{\partial \tilde{\mathbf{u}}}{\partial t}=\left\{-\nabla \cdot(\mathbf{u u})-\nabla \tilde{p}+\frac{1}{R e} \nabla^{2} \mathbf{u}+\frac{1}{F r^{2}} \mathbf{g}\right\},
$$

where $\tilde{p}$ is a provisional pressure. Generally, this provisional velocity field is not a solenoidal one, so $\tilde{p} \neq p$. For $t=t_{0}$, it is considered that both $\mathbf{u}\left(\mathbf{x}, t_{0}\right)$ and $\tilde{\mathbf{u}}\left(\mathbf{x}, t_{0}\right)$ satisfy the same boundary conditions and that on the boundary $\mathbf{u}\left(\mathbf{x}, t_{0}\right)=\tilde{\mathbf{u}}\left(\mathbf{x}, t_{0}\right)$. The main modification in the GENSMAC method is the inclusion of the Implicit Formulations $(I F)$ for two variations in the projection methods.

The first projection method is based on the solution of the time-discretized Equation (1.1), without a provisional pressure gradient (referred here as pressurefree projection method [5] and denoted by $P 1$ ), and the second projection method is based on the method with the provisional pressure gradient as in Equation (2.1) (referred to incremental-pressure projection methods [7] and denoted here by P2).

For the P1 method, it is used implicit methods for the viscous terms. The implicit schemes used were the Backward Implicit $(B I)$ and Crank-Nicolson $(C N)$ methods. In order to improve the temporal accuracy, a 2-step Adams method was employed. This method uses the $C N$ approximation for the viscous terms and the explicit Adams-Bashforth for the non-linear convective terms of Equation (2.1). This method is known as Adams-Bashforth/Crank-Nicolson $(A B / C N)$. Therefore, applying the method $P 1$ and using the implicit formulations, Equation (2.1) is rewritten in the following way

- P1 - BI method

$$
\tilde{\mathbf{u}}-\frac{\delta t}{R e} \nabla^{2} \tilde{\mathbf{u}}=\mathbf{u}^{n}+\delta t\left\{-\nabla \cdot(\mathbf{u u})^{n}+\frac{1}{F r^{2}} \mathbf{g}^{n}\right\} .
$$


- P1 - CN method

$$
\tilde{\mathbf{u}}-\frac{\delta t}{2 R e} \nabla^{2} \tilde{\mathbf{u}}=\mathbf{u}^{n}+\delta t\left\{-\nabla \cdot(\mathbf{u u})^{n}+\frac{1}{2 R e} \nabla^{2} \mathbf{u}^{n}+\frac{1}{F r^{2}} \mathbf{g}^{n}\right\} .
$$

- P1 - AB/CN method

$$
\tilde{\mathbf{u}}-\frac{\delta t}{2 R e} \nabla^{2} \tilde{\mathbf{u}}=\mathbf{u}^{n}+\delta t\left\{-\frac{3}{2} \nabla \cdot(\mathbf{u u})^{n}+\frac{1}{2} \nabla \cdot(\mathbf{u u})^{n-1}+\frac{1}{2 R e} \nabla^{2} \mathbf{u}^{n}+\frac{1}{F r^{2}} \mathbf{g}^{n}\right\} .
$$

Using the theory of the projection methods, we know that the tentative velocity $\tilde{\mathbf{u}}$ can be decomposed as

$$
\tilde{\mathbf{u}}=\mathbf{u}^{n+1}+\nabla \psi
$$

where $\mathbf{u}^{n+1}$ is divergence-free. In the $P 1$ method, the function $\psi$ is calculated in the whole domain.

In the same manner as in the method of $P 1$, the viscous terms were taken implicitly, and Equation (2.1) for P2 becomes

- P2 - BI method

$$
\tilde{\mathbf{u}}-\frac{\delta t}{R e} \nabla^{2} \tilde{\mathbf{u}}=\mathbf{u}^{n}+\delta t\left\{-\nabla \cdot(\mathbf{u u})^{n}-\nabla \tilde{p}+\frac{1}{F r^{2}} \mathbf{g}^{n}\right\}
$$

- P2 - CN method

$$
\tilde{\mathbf{u}}-\frac{\delta t}{2 R e} \nabla^{2} \tilde{\mathbf{u}}=\mathbf{u}^{n}+\delta t\left\{-\nabla \cdot(\mathbf{u u})^{n}+\frac{1}{2 R e} \nabla^{2} \mathbf{u}^{n}-\nabla \tilde{p}+\frac{1}{F r^{2}} \mathbf{g}^{n}\right\} .
$$

- P2 - $A B / C N$ method

$$
\begin{aligned}
& \tilde{\mathbf{u}}-\frac{\delta t}{2 R e} \nabla^{2} \tilde{\mathbf{u}}=\mathbf{u}^{n}+\delta t\left\{-\frac{3}{2} \nabla \cdot(\mathbf{u u})^{n}+\frac{1}{2} \nabla \cdot(\mathbf{u u})^{n-1}+\frac{1}{2 R e} \nabla^{2} \mathbf{u}^{n}-\nabla \tilde{p}\right. \\
& \left.+\frac{1}{F r^{2}} \mathbf{g}^{n}\right\} .
\end{aligned}
$$

The development of the $P 2$ method using $I F$ is similar to the $P 1$ method, with the difference that now $\tilde{p} \neq 0$ will be calculated. As the GENSMAC method, the Poisson equation for $\psi$ is applied for the whole domain containing fluid, with the appropriate boundary conditions described in [11]. For the $P 1$ and $P 2$ methods using $I F$, besides the Poisson equation, a new equation is imposed on the potential $\psi$ on the free surface. This new equation is calculated from the equation of the pressure in the free surface. On the free surface, it is necessary to impose conditions on the velocity and pressure. Considering absent surface tension, these conditions are summarized as

$$
(\mathbf{T} \cdot \mathbf{n}) \cdot \mathbf{n}=0 \quad \text { and } \quad(\mathbf{T} \cdot \mathbf{n}) \cdot \mathbf{m}=0
$$


where $\mathbf{n}=\left(n_{x}, n_{y}\right)$ is the external normal vector to the surface, and $\mathbf{m}=\left(m_{x}, m_{y}\right)$ is the tangent vector to the free surface. By substituting the total tensor $\mathbf{T}=-p \mathbf{I}+\boldsymbol{\tau}$, where $\boldsymbol{\tau}$ is the stress tensor and $\mathbf{I}$ the identity tensor, in Equation (2.9) we obtain

$$
\begin{gathered}
-p+\frac{2}{R e}\left[\frac{\partial u}{\partial x} n_{x}^{2}+\frac{\partial v}{\partial y} n_{y}^{2}+\left(\frac{\partial u}{\partial y}+\frac{\partial v}{\partial x}\right) n_{x} n_{y}\right]=0, \\
2 \frac{\partial u}{\partial x} n_{x} m_{x}+2 \frac{\partial v}{\partial y} n_{y} m_{y}+\left[\frac{\partial u}{\partial y}+\frac{\partial v}{\partial x}\right]\left(n_{y} m_{x}+n_{x} m_{y}\right)=0 .
\end{gathered}
$$

The equations for $\psi$ at the free surface are derived from the equation of the pressure Equation (2.10) with implicit velocity. The application of $P 1$ and $P 2$ methods for the implicit formulations results in 3 sparse linear systems: 2 due to the equations that calculate the intermediary velocity and 1 due to the calculation of the potential $\psi$. When the implicit formulations are applied, for the $B I$ or $C N$ methods, the viscous terms are taken implicitly, and for this it is necessary to solve systems for velocities $\tilde{u}$ and $\tilde{v}$. The linear systems resulting from Equations (2.2)-(2.8) are sparse, positive definite and symmetric. Due to those properties, an efficient method is the Conjugated Gradient (CG) method. The linear system for $\psi$ is sparse, but non-symmetric, and therefore the method used was the Bi-Conjugated Gradients with Preconditioning (BCGP). Besides the method BCGP, other alternatives are recommended in the literature for sparse problems: the GMRES (Generalized Minimum Residual) and PCGS (Preconditioned Conjugate Gradient Squared) are two examples. More details of the numerical methods using implicit formulations and boundary conditions at the free surfaces can be found in [9].

\subsection{Stability of $P 1$ and $P \mathscr{2}$ methods}

The stability restriction imposed for explicit treatment of the viscous terms requires that

$$
\delta t_{\text {viscous }} \leq 0.5 \operatorname{Re}\left[(\delta x)^{-2}+(\delta y)^{-2}\right]^{-1},
$$

where $\delta x$ and $\delta y$ are grid spacings. The application of Implicit Formulations for the viscous terms as in Equations (2.2)-(2.8), can, in principle, remove the restriction (2.12). Therefore, the restrictions on $\delta t$ for $P 1$ and $P \mathcal{2}$ using $I F$ are more relaxed than that of the original GENSMAC code.

\section{2. $\quad$ Solution procedure}

The sequence of steps in the solution procedure purports updating the discrete variables, starting from an initial time $t_{n}$. The algorithm is described as follow

- Step 1: For the P1 method, the pressure gradient $\nabla \tilde{p}$ is eliminated from the formulation and the velocity at the free surfaces is calculate from Equation (2.11). For the P2 method, the velocity at the free surfaces is normally computed, and the pressure gradient is conserved, that is $\tilde{p}=p^{n}$, where $p^{n}$ is the pressure calculated in the previous time from Equation (2.10); 
- Step 2: Calculate an intermediary velocity field $\tilde{\mathbf{u}}(\mathbf{x}, t)$ in $t=t_{n}+\delta t$. When the $P 1$ method is used, Equations (2.2), (2.3) and (2.4) can be used. Similarly, when the P2 method is applied, Equations (2.6), (2.7) and (2.8) can be used;

- Step 3: Solve the Poisson equation for the potential $\psi$ in regions that contain fluid, and at the free surfaces, $\psi$ is calculated from Equation (2.10) with implicit velocity. Details of the boundary conditions for the Poisson equation and the equations for $\psi$ at the free surfaces can be found in [9];

- Step 4: Compute the corrected velocity field $\mathbf{u}^{n+1}=\tilde{\mathbf{u}}-\nabla \psi^{n+1}$;

- Step 5: Compute the final pressure field. For the $P 1$ method, the pressure is computed from equation $p^{n+1}=\psi^{n+1} / \delta t$, and for the P2 method, the equation is $p^{n+1}=\tilde{p}+\psi^{n+1} / \delta t$;

- Step 6: Update the marker particles positions. The last step in the calculation is moving the marker particles to their new positions. This is performed by solving $\dot{x}=u$ and $\dot{y}=v$ by Euler's method. The fluid surface is defined by a list containing these particles and the visualization of this boundary is obtained by connecting them by straight lines.

\section{Discrete Equations}

Equations (1.1) and (1.2) are approximated in a staggered mesh. In this mesh, the pressure is stored at cell centers and the components of the velocity $u$ and $v$ are stored in the middle of the lateral faces. As in [11], the diffusion terms and the pressure gradient in Equations (2.2)-(2.8) are approximated by central differences, whereas the time derivatives are approximated by forward differences (Euler explicit). The convective terms are discretized by VONOS scheme (see [6]), which is a bounded upwind technique. For solving the conservation equations, the FREEFLOW2D (see [4]) simulation environment will be used. This system is composed of three module: a modeling module (modeler) a simulation module (simulator, which implements the full Navier-Stokes equations and mass conservation equation) and the visualization module (visualizator).

\section{Numerical Results}

In this section, numerical results using the implicit formulations are presented. The main aim is comparing $P 1$ and $P 2$ methods, using $B I, C N$ and $A B / C N$ formulations, with the explicit method for problems in which $R e<1$. In relation to the explicit method, the results are encouraging, in terms of accuracy and efficiency. The following test cases are considered.

\subsection{Hagen-Poiseuille flow}

The validation of the numerical results obtained by using $P 1$ and $P 2$ methods with $I F$ was performed for the flow between two parallel plates. In this test case, 
comparisons between the numerical solutions and analytic solution are feasible (see [2]). In this simulation, the plates are separated by a distance $L=1$, forming a channel. In the beginning, the channel is empty and the fluid is injected at the entrance region, using a parabolic velocity profile. The $P 1$ and $P 2$ methods using $I F$ were applied in three meshes defined respectively as coarse (M1, where $\delta x=\delta y=0.1 \mathrm{~m})$, middle $(\mathbf{M} 2$, where $\delta x=\delta y=0.05 \mathrm{~m})$, and fine (M3, where $\delta x=\delta y=0.025 \mathrm{~m})$ meshes. The results are in good agreement, on the three meshes, with the analytic solution. In order to show the convergence of the methods presented in this work, the relative error in the $l_{2}$ norm, between the numerical solutions and the analytic, was calculated. These results are presented in Table 1. One can see from this table that for creep flow problems, the $I F$ was more stable

Table 1: Results of $\delta t$ and error (Er) for Hagen-Poiseuille flow for $R e=0.1$ in the meshes M1, M2 and M3.

\begin{tabular}{|c|c|c|c|c|c|c|}
\hline \multirow{2}{*}{ Method } & \multicolumn{2}{|c|}{ M1 } & \multicolumn{2}{c|}{ M2 } & \multicolumn{2}{c|}{ M3 } \\
\cline { 2 - 7 } & $\delta t(\mathrm{~s})$ & $E r$ & $\delta t(\mathrm{~s})$ & $E r$ & $\delta t(\mathrm{~s})$ & $E r$ \\
\hline Explicit & $1.0 \times 10^{-4}$ & $2.5 \mathrm{E}-05$ & $2.5 \times 10^{-5}$ & $1.8 \mathrm{E}-06$ & $6.25 \times 10^{-6}$ & $1.3 \mathrm{E}-07$ \\
$P 1-B I$ & $1.25 \times 10^{-3}$ & $7.1 \mathrm{E}-04$ & $2.5 \times 10^{-4}$ & $3.7 \mathrm{E}-05$ & $6.25 \times 10^{-5}$ & $3.2 \mathrm{E}-06$ \\
$P 1-C N$ & $2.0 \times 10^{-3}$ & $5.6 \mathrm{E}-04$ & $5.0 \times 10^{-4}$ & $4.5 \mathrm{E}-05$ & $1.25 \times 10^{-4}$ & $3.2 \mathrm{E}-06$ \\
P1-AB/CN & $2.0 \times 10^{-3}$ & $1.6 \mathrm{E}-04$ & $5.0 \times 10^{-4}$ & $2.5 \mathrm{E}-05$ & $1.25 \times 10^{-4}$ & $2.1 \mathrm{E}-06$ \\
P2-BI & $1.0 \times 10^{-2}$ & $2.5 \mathrm{E}-05$ & $1.25 \times 10^{-2}$ & $1.8 \mathrm{E}-06$ & $6.25 \times 10^{-3}$ & $1.2 \mathrm{E}-07$ \\
P2-CN & $2.0 \times 10^{-3}$ & $2.5 \mathrm{E}-05$ & $5.0 \times 10^{-4}$ & $1.8 \mathrm{E}-06$ & $5.0 \times 10^{-4}$ & $1.1 \mathrm{E}-07$ \\
P2- $A B / C N$ & $2.0 \times 10^{-3}$ & $2.5 \mathrm{E}-05$ & $5.0 \times 10^{-4}$ & $1.8 \mathrm{E}-06$ & $5.0 \times 10^{-4}$ & $1.1 \mathrm{E}-07$ \\
\hline
\end{tabular}

than the original explicit method. Table 2 shows the $\delta t$ allowed by implicit and explicit formulations. The methods that use the formulation $B I$ admitted values of $\delta t$ larger than the other formulations. When the Re decreases, the restriction on the time step for the explicit method (2.12) was overcome by the $P 1$ and $P 2$ methods using $I F$. From Table 2, it can be seen that the methods using the formulation $B I$ demanded a $\delta t$ about 500 the 500000 larger times than the explicit method, when $R e$ decreases, while the formulations $C N$ and $A B / C N$ presented $\delta t$ about 20 times bigger, independent of Re.

Table 2: Limited of stability for $\delta t(\mathrm{~s})$ in Hagen-Poiseuille flow over the mesh M2, with values different for $R e$.

\begin{tabular}{|c||c||c||c||c|}
\hline Method & $R e=0.1$ & $R e=0.01$ & $R e=0.001$ & $R e=0.0001$ \\
\hline Explicit & $2.5 \times 10^{-5}$ & $2.5 \times 10^{-6}$ & $2.5 \times 10^{-7}$ & $2.5 \times 10^{-8}$ \\
\hline$P 1-B I$ & $1.25 \times 10^{-2}$ & $1.25 \times 10^{-2}$ & $1.25 \times 10^{-2}$ & $1.25 \times 10^{-2}$ \\
\hline$P 1-C N$ & $5.0 \times 10^{-4}$ & $5.0 \times 10^{-5}$ & $5.0 \times 10^{-6}$ & $5.0 \times 10^{-7}$ \\
\hline$P 1-A B / C N$ & $5.0 \times 10^{-4}$ & $5.0 \times 10^{-5}$ & $5.0 \times 10^{-6}$ & $5.0 \times 10^{-7}$ \\
\hline$P 2-B I$ & $1.25 \times 10^{-2}$ & $1.25 \times 10^{-2}$ & $1.25 \times 10^{-2}$ & $1.25 \times 10^{-2}$ \\
\hline$P \mathcal{2}-C N$ & $5.0 \times 10^{-4}$ & $5.0 \times 10^{-5}$ & $5.0 \times 10^{-6}$ & $5.0 \times 10^{-7}$ \\
\hline$P \mathcal{L}-A B / C N$ & $5.0 \times 10^{-4}$ & $5.0 \times 10^{-5}$ & $5.0 \times 10^{-6}$ & $5.0 \times 10^{-7}$ \\
\hline
\end{tabular}




\subsection{Container filling}

In this test case, it is considered the problem of container filling by a newtonian fluid with $R e=0.1$. In this simulation a comparison of CPU time was made using the $P 1$ and $P 2$ methods with $I F$, and the explicit method. A mesh $\delta_{x}=\delta_{y}=0.00050 \mathrm{~m}$ was used for all the methods. The gravitational field acts on the flow and the final time of the simulations was $t=5 \mathrm{~s}$. The results obtained by the $P 1$ method, using the $B I, C N$ and $A B / C N$ formulations and those by $P 2$ method using the $B I$ and $A B / C N$ formulations were very similar to those of the $P \mathscr{2}$ method using the $C N$ formulation. The comparison between the methods that use the implicit and explicit formulations, verifying the value of $\delta t$ allowed for each method, the number of iterations and the CPU time for the time $t=0.28 \mathrm{~s}$, can be seen in Table 3 . Again, the implicit formulations overcame the restriction of stability of the original explicit method. These methods used less iterations to obtain the solution at the time $t=0.28 \mathrm{~s}$.

Table 3: Results for simulation of container filling. Input data employed: $L=$ $0.05 \mathrm{~m}, U=1.0 \mathrm{~ms}^{-1}, R e=0.1$ and $t=0.28 \mathrm{~s}$.

\begin{tabular}{|c||c||c||c|}
\hline Method & $\delta t(\mathrm{~s})$ & Number of iteration & CPU time- $(\mathrm{m}: \mathrm{s})$ \\
\hline Explicit & $5.0 \times 10^{-7}$ & 559998 & $430: 59$ \\
\hline$P 1-B I$ & $3.0 \times 10^{-5}$ & 11200 & $41: 52$ \\
\hline$P 1-C N$ & $1.0 \times 10^{-5}$ & 28000 & $99: 16$ \\
\hline$P 1-A B / C N$ & $1.0 \times 10^{-5}$ & 28000 & $106: 18$ \\
\hline$P 2-B I$ & $6.0 \times 10^{-5}$ & 8960 & $21: 41$ \\
\hline$P 2-C N$ & $1.0 \times 10^{-5}$ & 28000 & $92: 51$ \\
\hline$P 2-A B / C N$ & $1.0 \times 10^{-5}$ & 28000 & $96: 25$ \\
\hline
\end{tabular}

\subsection{A qualitative comparison}

Finally, in this test case, qualitative comparisons between numerical results with the experiments described in [12] is assessed. For this model, a mesh of $\delta_{x}=\delta_{y}=$ $0.00050 \mathrm{~m}$ was used for all the methods, with the gravitational field acting on the flow and the final time was $t=5 \mathrm{~s}$. Figure 1 presents the comparison between the numerical solution and an experimental configuration. In this figures, the numerical method used was the $P 2$ method with the $B I$ formulation. The other methods that use the implicit formulations are not displayed because they presented results similar to the $P 2$ method using the formulation $B I$. The implicit formulations presented, as previously, larger values for $\delta t$, overcoming the restriction of the explicit method described by [12].

\section{Conclusion}

The main purpose of this work is a study of numerical schemes for solving twodimensional, time-dependent, viscous incompressible free surface flows. A modifi- 
a)
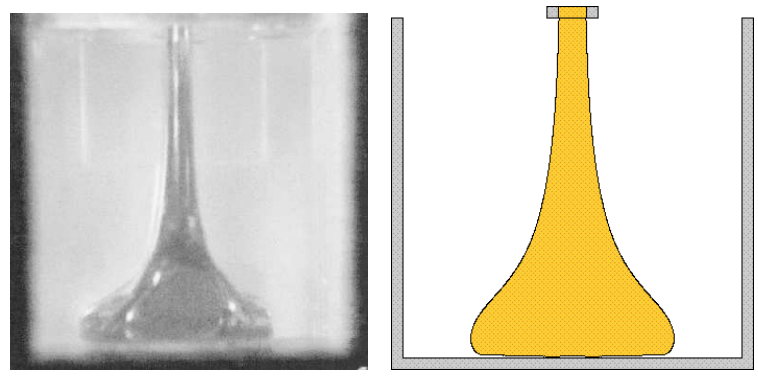

b)
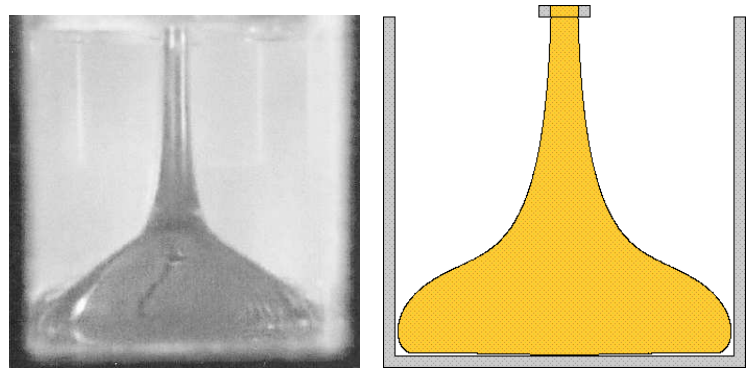

c)
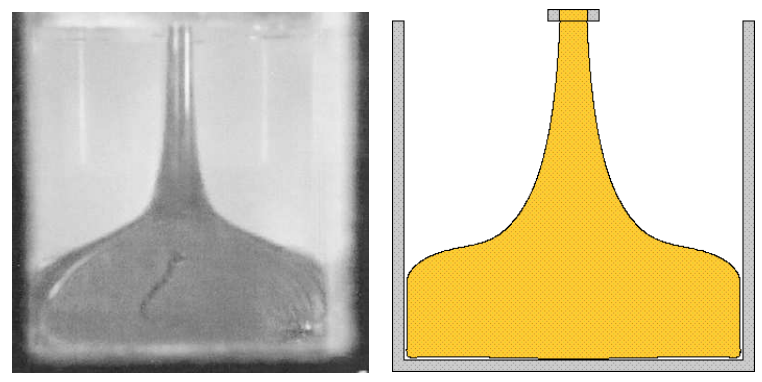

d)
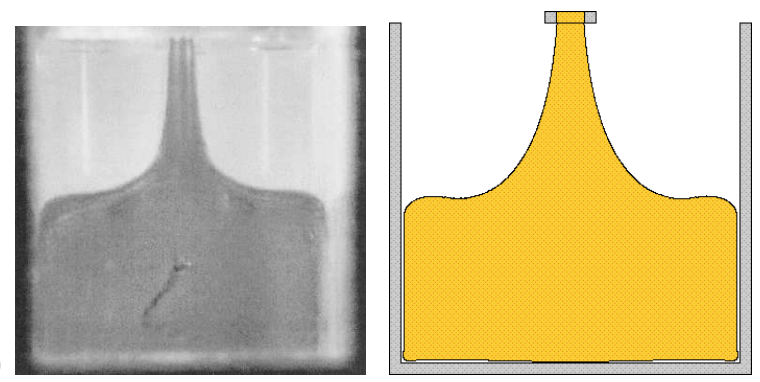

Figure 1: Experimental solution (left) and numerical solution (right). In this simulation, the $P 2$ method with the $B I$ formulation was used. a) $t=0.14 \mathrm{~s}, \mathbf{b}) t=0.22 \mathrm{~s}$, c) $t=0.26 \mathrm{~s}$ and $\mathbf{d}) t=0.34 \mathrm{~s}$. 
cation was made to the implicit treatment of boundary conditions for pressure at the free surface. The implicit formulations presented satisfactory results for unsteady free surface flows. The validation showed the comparison between the analytic solution and the numerical solution of the $P 1$ and $P 2$ methods using $I F$. The numerical results shows the capacity of this semi-implicit methods in simulate fluid flow with free surface. However, the $C N$ and $A B / C N$ formulations introduced numerical oscillations, and as a consequence, the value of $\delta t$ allowed was more restricted than that of the $B I$ formulation. More details about the numerical oscillations of the method $C N$ can be found in [13]. Although the $C N$ and $A B / C N$ formulations have allowed a time step larger than that of the original explicit method, the $B I$ formulation proved to be stable allowing values of $\delta t$ very large. Care is recommended in choosing the time step so that numerical accuracy is not affected. In all the simulations, the implicit formulations overcame the value of the time step of the explicit method and, in some cases, the $\delta t$ was approximately 500000 times larger than the one of the explicit method. The $P 1$ and $P 2$ methods using the implicit formulations presented similar errors to those of the explicit method with a very smaller number of iterations. The processing time demanded by the implicit formulations was significantly smaller than those of the explicit formulation. Therefore, the $P 1$ and $P 2$ methods using the implicit formulations showed to be capable of solving viscous problems with free surfaces.

Resumo. O presente trabalho concentra-se em um estudo de métodos numéricos para resolver escoamentos newtonianos bidimensionais, transientes, incompressíveis e com superfície livre. Um método de projeção é aplicado para desacoplar os campos de velocidade e de pressão. Os termos difusivos nas equações de Navier-Stokes são tratados implicitamente por meio das formulações Implícita Regressiva $(I R)$, Crank-Nicolson $(C N)$ e Adams-Bashforth/Crank-Nicolson $(A B / C N)$. Os termos advectivos são tratados explicitamente por um esquema upwind de alta ordem limitado. Para melhorar a estabilidade numérica, as condições de contorno para o campo de pressão na superfície livre são tratadas implicitamente, e para o campo de velocidade explicitamente. Os resultados numéricos mostram que o método elimina a restrição de estabilidade do método explícito original GENSMAC.

\section{Acknowledgments}

This work was financially supported by FAPESP, contracts 03/12612-9, 01/12540-2 and 00/03385-0.

\section{References}

[1] S. Armfield and R. Street, The pressure accuracy of fractional-step methods for the Navier-Stokes equations on staggered grids, ANZIAM. Australian and New Zealand Industrial and Applied Mathematics, 44 (2003), 20-39.

[2] G.K. Batchelor, "An Introduction to Fluid Dynamics", Cambridge, 1970.

[3] W.R. Briley and H. McDonald, An overview and generalization of implicit Navier-Stokes algorithms and approximate factorization, Comput. \& Fluid, 30 (2001), 807-828. 
[4] A.F. Castelo, M.F. Tomé, M.L. Cesar, J.A. Cuminato and S. Mckee, Freeflow: An intregated simulation system for three-dimensional free surface flows, Journal of computers and visualization in science, 2 (2000), 199-210.

[5] A.J. Chorin, A numerical method for solving incompressible viscous flow problems, Journal of Computational Physics, 2 (1967), 12-26.

[6] V.G. Ferreira, M.F. Tomé, N. Mangiavacchi, A. Castelo, J.A. Cuminato and S. McKee, High order upwinding and the hydraulic jump, Int. J. Numer. Meth. Fluid, 39 (2002), 549-583.

[7] P.M. Gresho, On the theory of semi-implicit projection methods for viscous incompressible flow and its implemention via a finite element method that also introduces a nearly consistent mass matrix, International Journal for Numerical Methods in Fluids, 11 (1990), 587-620.

[8] M. Manna and A. Vacca, An efficient method for the solution of the Incompressible Navier-Stokes equations in cylindrical geometries, Journal of Computational Physics, 151 (1999), 563-584.

[9] C.M. Oishi, "Análise e implementação de métodos implícitos no sistema FreeFlow2D”, Master's Thesis, ICMC, University of São Paulo, 2004.

[10] P.J. Roache, "Fundamental of Computational Fluid Dynamics", Albuquerque, Hermosa Publishers, 1998.

[11] M.F. Tomé and S. McKee, GENSMAC: A computational marker-and-cell method for free surface flows in general domains, J. Comput. Phys., 110 (1994), 171-186.

[12] M.F. Tomé, S. McKee, L. Barratt, D.A. Jarvis and A.J. Patrick, An experimental and numerical investigations of container filling with viscous liquids, International Journal for Numerical Methods in Fluids, 31 (1999), 1333-1353.

[13] S. Turek, A comparative study of some time-stepping techniques for the incompressible Naveir-Stokes equations: From fully implicit nonlinear schems to semi-implicit projection methods, International Journal for Numerical Methods in Fluids, 22 (1996), 987-1011. 